Meeting report

\title{
Allogeneic peripheral blood stem cell transplantation
}

D. Blaise

IPC, 232, Boulevard Ste. Marguerite, BP 156, F-13273 Marseille Cedex 09, France

Recently the development of Allo PBSC transplantation has followed the promising results obtained in Autologous setting for hematological reconstitution. However this setting raised numerous questions: Is long term engrafment obtained? Is the reinfusion of T cells in large number associated with the modification of post-graft immune reactions? Collection needs a priming with an hematopoietic growth factor: what is the impact on a normal donor both at short and long follow-up?

None of these questions are definitively solved:

- when engrafment occurs, it seems that platelet reconstitution is fastened, however in several occasions, graft failure has been reported. It is yet difficult to distinguish between patients' selection for a pilot study and real medical problem;

- a large number of T lymphocytes is not systematically associated with AGVHD, raising some questions about the functions of primed T-cells. However, it seems definitively clear that this technology is associated with an increase rate of chronic GVHD;

- the study of donor has very often been neglected in Allo BMT. However, the use of general anesthesia is not without risk. The use of an harvest not needing this general anesthesia is appealing, however, little is known about the long term effect of HGF in normal donors and this needs a prospective evaluation.

After a pilot study of 61 patients which we conducted in France, the preliminary analysis of which will be presented at the SFGM congress, we decided to conduct a prospective trial comparing Allo PBSCT and BMT. This trial is currently under process.

\section{Back to the HCT-EE Home Page}

Last change: April 28, 1997

helpdesk.link@springer.de

(C) 1996 by Springer-Verlag France 